\title{
Analysis of excreta bacterial community after forced molting in aged laying hens
}

\author{
Gi Ppeum Han ${ }^{1, a}$, Kyu-Chan Lee ${ }^{2, a}$, Hwan Ku Kang ${ }^{3}$, Han Na Oh², Woo Jun Sul ${ }^{2, *}$, and Dong Yong Kil ${ }^{1, *}$
}

\author{
* Corresponding Authors: \\ Woo Jun Sul \\ Tel: +82-31-670-4707, Fax: +82-31-675-3108 \\ E-mail: sulwj@cau.ac.kr \\ Dong Yong Kil \\ Tel: +82-31-670-3028, Fax: +82-31-675-3108 \\ E-mail: dongyong@cau.ac.kr \\ 1 Department of Animal Science and Technology, \\ Chung-Ang University, Anseong 17546, Korea \\ 2 Department of Systems Biotechnology, Chung-Ang \\ University, Anseong 17546, Korea \\ ${ }^{3}$ Poultry Research Institute, National Institute of \\ Animal Science, Rural Development Administration, \\ Pyeongchang 25342, Korea \\ a These authors contributed equally to this work. \\ ORCID \\ Gi Ppeum Han \\ https://orcid.org/0000-0001-7794-2213 \\ Kyu-Chan Lee \\ https://orcid.org/0000-0002-3235-7798 \\ Hwan Ku Kang \\ https://orcid.org/0000-0002-4286-3141 \\ Han Na Oh \\ https://orcid.org/0000-0002-6174-4359 \\ Woo Jun Sul \\ https://orcid.org/0000-0002-7016-1454 \\ Dong Yong Kil \\ https://orcid.org/0000-0002-9297-849X
}

Submitted Mar 6, 2019; Revised May 13, 2019; Accepted May 29, 2019
Objective: As laying hens become aged, laying performance and egg quality are generally impaired. One of the practical methods to rejuvenate production and egg quality of aged laying hens with decreasing productivity is a forced molting. However, the changes in intestinal microbiota after forced molting of aged hens are not clearly known. The aim of the present study was to analyze the changes in excreta bacterial communities after forced molting of aged laying hens.

Methods: A total of one hundred 66-wk-old Hy-Line Brown laying hens were induced to molt by a 2-d water removal and an 11-d fasting until egg production completely ceased. The excreta samples of 16 hens with similar body weight were collected before and immediately after molting. Excreta bacterial communities were analyzed by high-throughput sequencing of bacterial 16S rRNA genes.

Results: Bacteroidetes, Firmicutes, and Proteobacteria were the three major bacterial phyla in pre-molting and immediate post-molting hens, accounting for more than 98.0\%. Lactobacillus genus had relatively high abundance in both group, but decreased by molting $(62.3 \%$ in premolting and 24.9\% in post-molting hens). Moreover, pathogenic bacteria such as Enterococcus cecorum and Escherichia coli were more abundant in immediate post-molting hens than in pre-molting hens. Forced molting influenced the alpha diversity, with higher Chaol $(\mathrm{p}=$ $0.012)$, phylogenetic diversity whole tree $(p=0.014)$, observed operational taxonomic unit indices $(p=0.006)$, and Simpson indices $(p<0.001)$, which indicated that forced molting increased excreta bacterial richness of aged laying hens.

Conclusion: This study improves the current knowledge of bacterial community alterations in the excreta by forced molting in aged laying hens, which can provide increasing opportunity to develop novel dietary and management skills for improving the gastrointestinal health of aged laying hens after molting.

Keywords: Aged Laying Hen; Excreta Bacterial Community; Forced Molting; High-throughput $16 \mathrm{~S}$ rRNA Sequencing

\section{INTRODUCTION}

As laying hens are aged, laying performance and egg quality are generally impaired [1]. One of the commercial solutions to restore performance and egg quality of aged laying hens is to induce the molting of hens because molting hens allows their reproductive organ to rest temporarily such that egg production and egg quality are recovered [2]. Thus, the current poultry industry in many countries adopts various molting methods including the temporary withdrawal of feeds and water and feeding unconventional diets containing excessive minerals (e.g., $\mathrm{Zn}$ and $\mathrm{Al}$ ), low nutritive ingredients (e.g., high fiber ingredients), or inadequate $\mathrm{Ca}$ [3]. Among these methods, forced molting induced by the temporary withdrawal of feeds and water has been widely practiced because of the low cost and promising results. 
However, animal welfare issues are often raised with the methods of forced molting.

In addition to animal welfare issues, the conventional forced molting method is known to increase the susceptibility of hens to various pathogenic bacteria, especially Salmonella enteritidis (SE), and accordingly, increases the possibility of Salmonella contamination in eggs, which is the greatest concern on eggrelated foodborne illness [4]. Various techniques including dietary modifications with alternative feed ingredients or additives have been evaluated to reduce the risk of Salmonella contamination in eggs [5]; however, no techniques have been satisfactory. One possible reason for unsatisfactory results for various strategies may be attributed to limited information regarding how molting procedures change microbial populations in the gastrointestinal tract (GIT), which directly influences SE colonization of hens and eggs.

Most of the current knowledge of microbial populations in poultry has been established based on classical culture techniques. However, these techniques can identify only a small part of total microbial populations because of inherent limitation to discover individual microbiota [6]. Recently, cultureindependent methods such as DNA sequencing techniques (e.g., next generation sequencing) enable us to analyze the entire microbial genome from specific areas of interest in a short time. This technique can provide massive sequencing data, which allows researchers to obtain thorough knowledge of microbial composition, structure, and diversity [7].

Therefore, the objective of the current study was to explore microbial changes in the GIT due to molting by comparing excreta bacterial communities in pre-molting vs. immediate post-molting laying hens.

\section{MATERIALS AND METHODS}

\section{Animals, experimental design, and diet}

All experimental procedures were reviewed and approved by the Animal Care and the Use Committee at Chung-Ang University. A total of one hundred 66-wk-old Hy-Line Brown laying hens were initially used in the current experiment. All hens were fed a commercial layer diet $(2,774 \mathrm{kcal} / \mathrm{kg}$ nitrogencorrected apparent metabolizable energy $\left[\mathrm{AME}_{\mathrm{n}}\right], 15.14 \%$ crude protein $[\mathrm{CP}], 3.75 \% \mathrm{Ca}$, and $0.32 \%$ available $\mathrm{P}$ ) and the average egg production rate was $82 \%$ before the start of molting. All hens were placed individually in cages $(30 \mathrm{~cm} \times 37 \mathrm{~cm}$ $\times 40 \mathrm{~cm}$, width $\times$ length $\times$ height) with a plastic tray hanging under the cage for the excreta collection. Before forced molting procedure was started at 3:30 pm, excreta droppings from individual hens were continuously monitored, collected aseptically in a $15-\mathrm{mL}$ tube, and frozen with liquid nitrogen. If cecal contents were appeared, they were removed before excreta collection. A total of one hundred excreta samples were stored at $-80^{\circ} \mathrm{C}$ before analysis. Forced molting was induced by an 11-d fasting with an initial 2-d water removal until egg production completely ceased. Hens were exposed to $8 \mathrm{~h}$ of light:16 h of dark (8 L:16 D) during the molting period. The average room temperature was set at $18.4^{\circ} \mathrm{C}$ and the humidity was set at $45.9 \%$. The termination time of forced molting was determined when the average percentage of body weight (BW) loss in all hens reached approximately $20 \%$. The average BW of hens were $2.14 \mathrm{~kg}$ and $1.66 \mathrm{~kg}$ in the pre-molting and immediate post-molting group, respectively. After forced molting was completed, a total of sixteen laying hens with similar BW that was close to average BW of molted hens were selected again. Each hen consumed $40 \mathrm{~g}$ of a commercial layer diet $(2,870 \mathrm{kcal} / \mathrm{kg} \mathrm{AME}, 16.0 \% \mathrm{CP}, 1.80 \% \mathrm{Ca}$, and $0.34 \%$ available $\mathrm{P}$ ) immediately after terminating forced molting in order to facilitate the defecation. Excreta collection was performed using the same procedure for pre-molting hens. The microbial analysis was performed with the same hens $(n=16)$ before and after molting to study longitudinal changes in excreta bacterial communities by molting.

\section{DNA extraction and illumina miseq sequencing}

Approximately $0.25 \mathrm{~g}$ of excreta samples were used for metagenomic DNA extraction with the PowerFecal DNA Isolation Kit (MO BIO Laboratories Inc., Carlsbad, CA, USA) following the manufacture's protocol. The concentrations of extracted DNA were measured by Nanodrop 2000 spectrophotometer (Thermo Fisher Scientific, Wilmington, DE, USA). The polymerase chain reaction (PCR) amplification was performed using the primer targeting V4-V5 regions of the $16 \mathrm{~S}$ rRNA genes (forward: 5'-CCA GCA GCY GCG GTR AN-3'; reverse: 5'-CCG TCA ATT CNT TTR AGT-3'). The PCR conditions were performed as followed: $95^{\circ} \mathrm{C}$ for $3 \mathrm{~min}$ for denaturation, 33 cycles of amplification $\left(30 \mathrm{~s}\right.$ at $95^{\circ} \mathrm{C}, 30 \mathrm{~s}$ at $55^{\circ} \mathrm{C}$, and 1 min at $72^{\circ} \mathrm{C}$ ), and final elongation at $72^{\circ} \mathrm{C}$ for $5 \mathrm{~min}$. The product was barcoded with Nextera XT Index Kit v2 (Illumina, San Diego, CA, USA) and amplification of the barcoded DNA was carried for 8 cycles. Then, PCR amplicons were purified using the AMPure XP beads. The quality of the final product was evaluated with the Nanodrop 2000 spectrophotometer. These amplicons were sequenced using Illumina Miseq platform at the Macrogen Inc. (Seoul, Korea). The 16S rRNA gene sequences were uploaded to the NCBI's sequence read archie (SRA) with the accession no. SRR7244747.

\section{Bacterial community analysis}

The resulting sequences generated from Illumina MiSeq sequencer were analyzed with QIIME (ver. 1.9.1; QIIME development team, Boulder, CO, USA). The raw sequencing data were trimmed and merged using the python scripts iu-merge-pairs. Merged sequences were clustered into 97\% similarity operational taxonomic units (OTUs) using the UCLUST algorithm [8]. Relative abundance of taxonomic 
origin was calculated and summarized up to species level. The OTU sequences were rarefied for the measurement of alpha diversity. Chaol (bacterial richness), observed OTUs (counts of OTUs found in each sample), phylogenetic diversity (PD) whole tree (total length of phylogenetic branches), and Simpson indices (evenness) were used. Principal coordinate analysis ( $\mathrm{PCoA}$ ) based on the Jaccard distance was conducted for the comparison of excreta bacterial communities between pre-molting and immediate post-molting groups. Statistical significance between groups was assessed by the analysis of similarity with 999 permutations [9].

A linear discriminant effect size (LEfSe) analysis determined the bacteria that represent significant differences between pre-molting and immediate post-molting groups [10]. The threshold for the logarithmic linear discriminant analysis (LDA) score was set to 3.0. Statistical assessment was performed with non-parametric factorial Kruskal-Wallis sumrank test ( $\alpha=0.05)$, which tested significant differential abundance in the groups.

For the sequence comparison with pathogens in poultry, 14 pathogen sequences were downloaded from NCBI (Supplementary Table S1). The OTU sequences assigned as Escherichia coli (E. coli) and Enterococcus cecorum (E. cecorum) were extracted from the representative OTU sequences. The phylogenetic trees were constructed by the neighbor-joining method with MEGA 7.0 [11].

\section{RESULTS AND DISCUSSION}

\section{Analysis of excreta bacterial community}

Bacterial communities in the excreta of 16 hens at pre-molting and immediate post-molting times were analyzed based on $16 \mathrm{~S}$ rRNA gene sequencing on the V4-V5 region. A total of 5,062,766 paired-end reads from Illumina Miseq platform were merged into 1,773,312 reads (average 55,416 merged reads per sample) for the analysis. The sequences were clustered into 8,481 OTUs and taxonomically identified up to the species level. A total of 25 phyla, 63 classes, 114 orders, 212 families, 428 genera, and 556 species were obtained. Firmicutes, Bacteroidetes, and Proteobacteria were the three major bacterial phyla in pre-molting and immediate post-molting hens, accounting for more than $98 \%$ of phyla (Table 1). The other phyla including Fusobacteria and Actinobacteria were also detected (the data was not shown), but the abundance of these bacteria was less than $1.0 \%$. This result is in agreement with previous findings that Firmicutes, Bacteroidetes, and Proteobacteria were the 3 major bacterial phyla in the excreta sample of laying hens $[12,13]$. Among the major phyla, Firmicutes dominated both groups. The abundance of Firmicutes in the excreta as observed in the current experiment $(85.0 \%$ on average) was similar to the value ( $86.6 \%$ on average) reported by Videnska et al [14], but was higher than the values reported in other studies $[12,15]$.

In the current experiment, post-molting hens exhibited decreased abundance of Firmicutes (75.2\%) but increased abundance of Bacteroidetes (12.8\%) and Proteobacteria (11.3\%) as compared to pre-molting hens $(85.0 \%, 9.5 \%$, and $3.9 \%$ for Firmicutes, Bacteroidetes, and Proteobacteria, respectively). This community change in post-molting hens may represent microbial dysbiosis in the GIT of hens after molting. It has been reported that a higher ratio of Bacteroidetes to Firmicutes in the GIT was related to decreasing production of short chain fatty acids (SCFA) [16]. The decreased production of SCFA in the lower part of the GIT has been reported to impair intestinal health by lowering energy supply to intestinal cells, increasing luminal $\mathrm{pH}$, and decreased intestinal barrier functions $[13,17]$.

Abundant genus affiliated to Proteobacteria was varied between groups. For example, Psychrobacter (2.2\%; Pseudomanadales) were the major in pre-molting hens, whereas Escherichia showed predominance (10.8\%; Enterobacteriales) in immediate post-molting hens. At the genus level, Lactobacillus dominated in both groups, but its abundance was largely decreased after forced molting $(62.3 \%$ and $24.9 \%$ in pre-molting and immediate post-molting hens, respectively). Lactobacillus has been known as one of the most abundant genus in the GIT of poultry and humans [18]. Previous studies demonstrated that some species of Lactobacillus are related to improvements in animal performance $[19,20]$. Decreasing BW has been associated with a lower concentration of Lactobacillus in human [21]. Therefore, we supposed that a decrease in Lactobacillus could be associated with decreased BW of laying hens during fasting. In addition, the advantage of intestinal colonization of Lactobacillus spp. has been reported to prevent pathogenic colonization by lowering the $\mathrm{pH}$ of the GIT [22]. Thus, decreased populations in Lactobacillus spp. after molting as observed in this study may indicate a favorable environment for the pathogen colonization in the GIT, and thus, may allow more pathogens to be colonized in the GIT [23]. This speculation is also supported by our current findings that possible pathogenic species such as E. cecorum (22.2\%) and E. coli (10.8\%) were discovered more in immediate post-molting hens than in premolting hens.

\section{Pathogenic bacteria in post-molting hens}

To understand phylogenetic differences between the pathogens in poultry and the taxonomically assigned OTUs in this experiment, 14 pathogen sequences were used (Supplementary Table S1). A total of 242 and 281 representative sequences assigned to E. coli and E. cecorum, respectively, were aligned with 14 pathogen sequences.

The phylogenetic tree of OTUs for E. cecorum illustrated that $B$. cereus, S. aureus, and L. monocytogenes were clustered 
Table 1. Relative abundance ${ }^{1)}$ of top 9 bacterial taxa in the excreta between pre-molting and immediate post-molting hens

\begin{tabular}{|c|c|c|c|c|c|}
\hline Group & Phylum (\%) & Class (\%) & Order (\%) & Family (\%) & Genus (\%) \\
\hline \multirow[t]{9}{*}{ Pre-molting } & $\begin{array}{c}\text { Bacteroidetes } \\
(9.51 \pm 6.0)\end{array}$ & $\begin{array}{l}\text { Bacteroidia } \\
(9.44 \pm 6.0)\end{array}$ & $\begin{array}{c}\text { Bacteroidales } \\
(9.44 \pm 6.0)\end{array}$ & $\begin{array}{c}\text { Bacteroidaceae } \\
(3.97 \pm 2.5)\end{array}$ & $\begin{array}{l}\text { Bacteroides } \\
(3.97 \pm 2.5)\end{array}$ \\
\hline & & & & $\begin{array}{c}\text { Prevotellaceae } \\
(1.18 \pm 0.7)\end{array}$ & $\begin{array}{l}\text { Prevotella } \\
(1.18 \pm 0.7)\end{array}$ \\
\hline & & & & $\begin{array}{l}\text { Unclassified } \\
(1.39 \pm 0.9)\end{array}$ & $\begin{array}{l}\text { Unclassified } \\
(1.39 \pm 0.9)\end{array}$ \\
\hline & $\begin{array}{l}\text { Firmicutes } \\
(84.96 \pm 6.9)\end{array}$ & $\begin{array}{c}\text { Bacilli } \\
(68.60 \pm 8.1)\end{array}$ & $\begin{array}{l}\text { Lactobacillales } \\
(66.89 \pm 8.2)\end{array}$ & $\begin{array}{c}\text { Enterococcaceae } \\
\quad(4.10 \pm 2.1)\end{array}$ & $\begin{array}{c}\text { Enterococcus } \\
(3.22 \pm 2.0)\end{array}$ \\
\hline & & & & $\begin{array}{c}\text { Lactobacillaceae } \\
(62.32 \pm 8.3)\end{array}$ & $\begin{array}{l}\text { Lactobacillus } \\
(62.34 \pm 8.3)\end{array}$ \\
\hline & & & $\begin{array}{c}\text { Turicibacterales } \\
(1.63 \pm 0.5)\end{array}$ & $\begin{array}{l}\text { Turicibacteraceae } \\
\quad(1.63 \pm 0.5)\end{array}$ & $\begin{array}{l}\text { Turicibacter } \\
(1.63 \pm 0.5)\end{array}$ \\
\hline & & Clostridia $(15.93 \pm 4.2)$ & $\begin{array}{c}\text { Clostridiales } \\
(15.93 \pm 4.2)\end{array}$ & $\begin{array}{c}\text { Clostridiaceae } \\
(3.61 \pm 1.8)\end{array}$ & $\begin{array}{l}\text { Clostridium } \\
(3.50 \pm 1.8)\end{array}$ \\
\hline & & & & $\begin{array}{c}\text { Peptostreptococcaceae } \\
(7.49 \pm 3.7)\end{array}$ & $\begin{array}{l}\text { Unclassified } \\
(7.30 \pm 3.7)\end{array}$ \\
\hline & $\begin{array}{c}\text { Proteobacteria } \\
(3.90 \pm 3.3)\end{array}$ & $\begin{array}{c}\text { Gammaproteobacteria } \\
(3.57 \pm 3.3)\end{array}$ & $\begin{array}{c}\text { Pseudomanadales } \\
\quad(3.46 \pm 3.3)\end{array}$ & $\begin{array}{c}\text { Moraxellaceae } \\
(3.44 \pm 3.3)\end{array}$ & $\begin{array}{c}\text { Psychrobacter } \\
(2.20 \pm 2.1)\end{array}$ \\
\hline \multirow[t]{9}{*}{ Post-molting } & $\begin{array}{l}\text { Bacteroidetes } \\
(12.81 \pm 5.8)\end{array}$ & $\begin{array}{c}\text { Bacteroidia } \\
(12.72 \pm 5.8)\end{array}$ & $\begin{array}{l}\text { Bacteroidales } \\
(12.72 \pm 5.8)\end{array}$ & $\begin{array}{c}\text { Bacteroidaceae } \\
(5.48 \pm 2.6)\end{array}$ & $\begin{array}{l}\text { Bacteroides } \\
(5.48 \pm 2.6)\end{array}$ \\
\hline & & & & $\begin{array}{c}\text { Paraprevotellaceae } \\
(2.47 \pm 1.2)\end{array}$ & $\begin{array}{l}\text { Unclassified } \\
(2.30 \pm 1.1)\end{array}$ \\
\hline & $\begin{array}{l}\text { Firmicutes } \\
(75.17 \pm 7.2)\end{array}$ & $\begin{array}{c}\text { Bacilli } \\
(53.20 \pm 7.9)\end{array}$ & $\begin{array}{c}\text { Lactobacillales } \\
(49.53 \pm 7.7)\end{array}$ & $\begin{array}{c}\text { Enterococcaceae } \\
(22.26 \pm 5.9)\end{array}$ & $\begin{array}{l}\text { Enterococcus } \\
(22.24 \pm 5.9)\end{array}$ \\
\hline & & & & $\begin{array}{c}\text { Lactobacillaceae } \\
(25.00 \pm 6.0)\end{array}$ & $\begin{array}{l}\text { Lactobacillus } \\
(24.94 \pm 6.0)\end{array}$ \\
\hline & & & $\begin{array}{c}\text { Turicibacterales } \\
(3.50 \pm 0.7)\end{array}$ & $\begin{array}{l}\text { Turicibacteraceae } \\
\quad(3.50 \pm 0.7)\end{array}$ & $\begin{array}{l}\text { Turicibacter } \\
(3.50 \pm 0.7)\end{array}$ \\
\hline & & $\begin{array}{c}\text { Clostridia } \\
(21.41 \pm 3.2)\end{array}$ & $\begin{array}{c}\text { Clostridiales } \\
(21.41 \pm 3.2)\end{array}$ & $\begin{array}{c}\text { Clostridiaceae } \\
(4.60 \pm 1.4)\end{array}$ & $\begin{array}{l}\text { Clostridium } \\
(3.56 \pm 0.9)\end{array}$ \\
\hline & & & & $\begin{array}{l}\text { Lachnospiraceae } \\
(4.20 \pm 1.4)\end{array}$ & $\begin{array}{c}\text { Ruminococcus } \\
(2.16 \pm 1.0)\end{array}$ \\
\hline & & & & $\begin{array}{c}\text { Peptostreptococcaceae } \\
(5.88 \pm 1.8)\end{array}$ & $\begin{array}{l}\text { Unclassified } \\
(5.36 \pm 1.6)\end{array}$ \\
\hline & $\begin{array}{c}\text { Proteobacteria } \\
(11.3 \pm 4.4)\end{array}$ & $\begin{array}{c}\text { Gammaproteobacteria } \\
(11.12 \pm 4.4)\end{array}$ & $\begin{array}{c}\text { Enterobacteriales } \\
\quad(10.85 \pm 4.4)\end{array}$ & $\begin{array}{c}\text { Enterobacteriaceae } \\
(10.85 \pm 4.4)\end{array}$ & $\begin{array}{l}\text { Escherichia } \\
(10.78 \pm 4.4)\end{array}$ \\
\hline
\end{tabular}

"Relative abundance indicated as mean percentages \pm standard error (SE).

into 9 OTUs (Figure 1a). In the case of the phylogenetic tree of E. coli OTU sequences, three Salmonella spp. (Salmonella enterica, Salmonella gallinarum, and Salmonella typhimurium) and Aeromonas hydrophila were closely clustered. In particular, E. coli sequences were included in the same cluster with OTU sequence such as OTU15802 that was assigned to E. coli (Figure 1b).

It can be suggested, therefore, that forced molting may provide increasing opportunity for the colonization of pathogenic bacteria such as E. cecorum and E. coli. This result that pathogenic proliferation was increased after molting is consistent with previous studies [24]. In particular, E. cecorum was found to proliferate after molting, and was regarded as a pathogen, leading to an economical loss to poultry farmers [25].

Diversity and variation of excreta bacterial community For the estimation of alpha diversity, we rarefied the data to
3,515 sequences per sample and used various indices provided by QIIME pipeline. The bacterial diversity generally measures how many different species are present and how uniformly they are distributed according to the diversity index.

The result revealed significant differences in bacterial richness and diversity between pre-molting and immediate postmolting hens (Figure 2). Alpha diversity indices including Chaol, PD whole tree, observed OTUs, and Simpson index were increased after molting. These results indicate that forced molting may increase bacterial richness and diversity in the GIT of hens. Similar results for increased bacterial richness and diversity were also observed for fasting humans [26]. In addition, stressful conditions such as heat stress were reported to increase microbial diversity in chickens [27]. In contrast, the high diversity in bacterial communities has been often considered as a biological indicator of healthy and stable microbial populations in the GIT [13], which was not the case 


\section{a. Enterococcus cecorum OTUs}

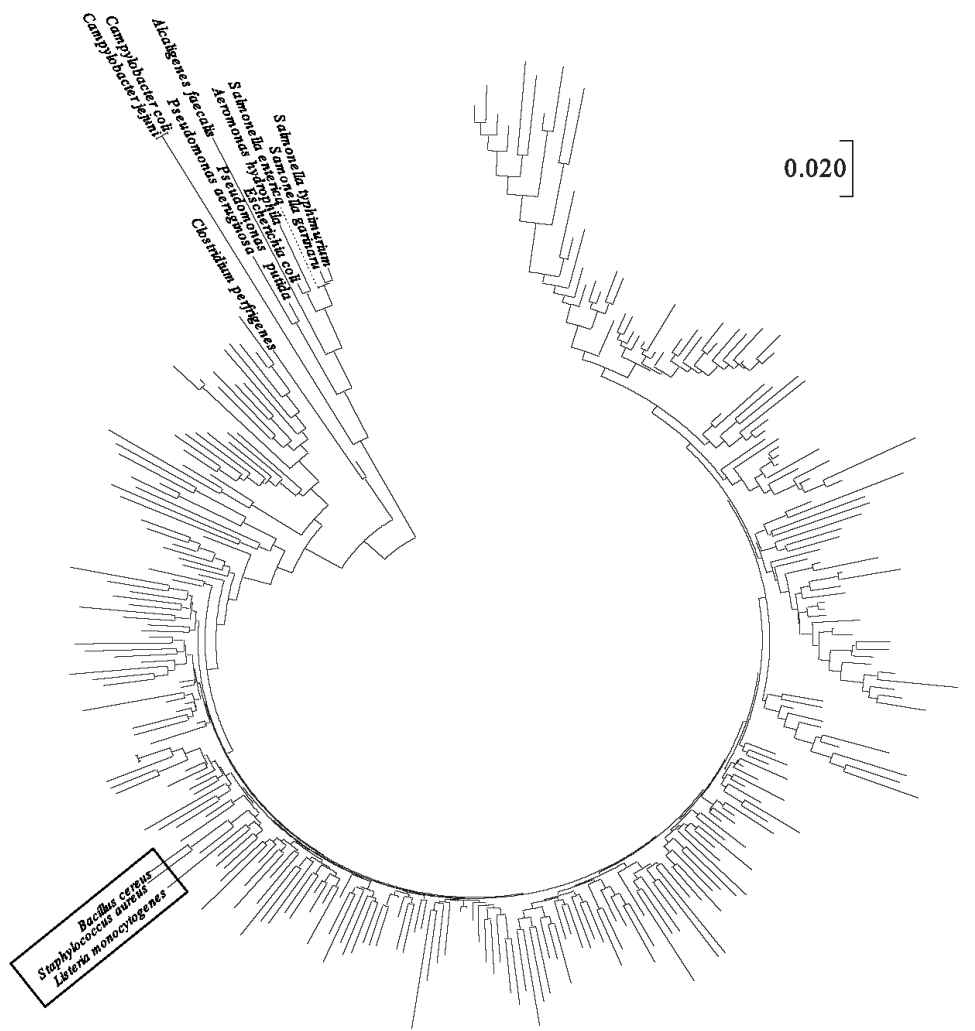

b. Escherichia coli OTUs

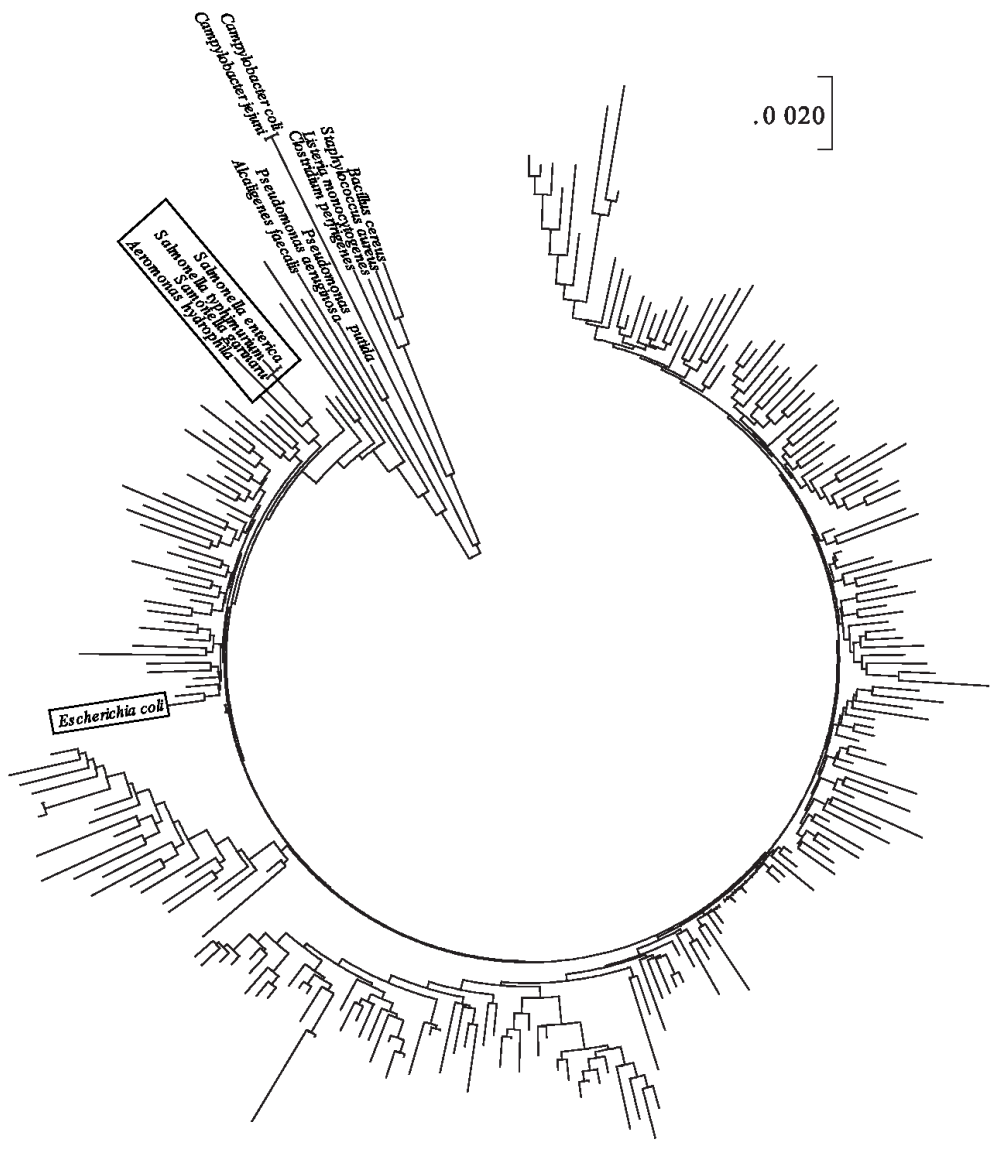

Figure 1. Phylogenetic trees constructed using the neighbor-joining method based on the nucleotide sequences of 14 pathogens. Operational taxonomic units sequences were assigned to (a) Enterococcus cecorum and (b) Escherichia coli. The phylogenetic tree was constructed from MEGA 7.0. 

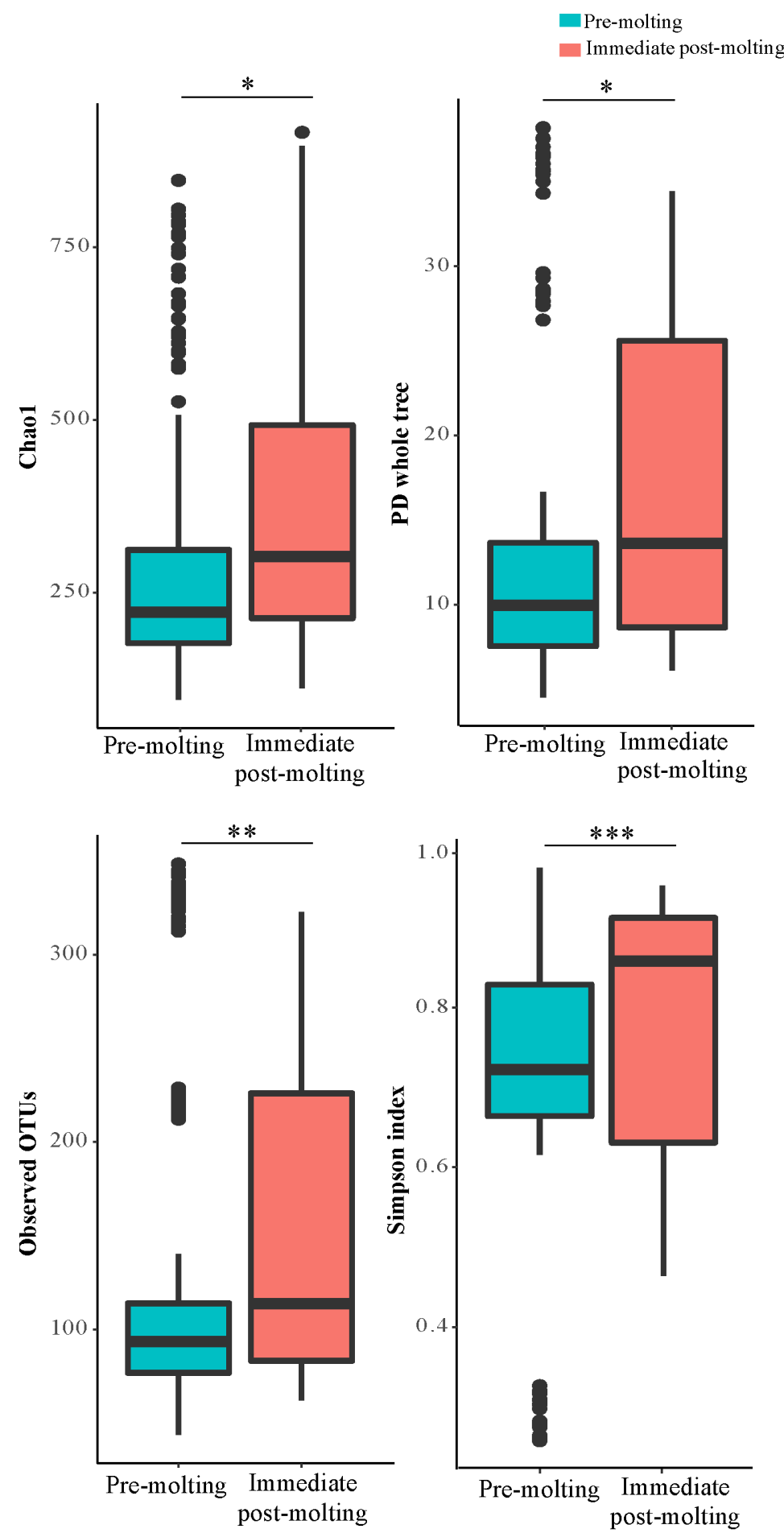

Figure 2. Alpha diversity: Choa1, PD whole tree, observed operational taxonomic units, and simpson indices in the excreta of pre-molting and immediate post-molting hens. For all 4 indices, higher values correspond to greater diversity. $p$-values were estimated by Wilcoxon test, indicating ${ }^{*} p<0.05,{ }^{* *} p<0.01,{ }^{* * *} p<0.001$.

in post-molting laying hens in the current experiment. Therefore, it is likely that increased bacterial richness and diversity cannot always have a direct association with healthy environment in the GIT. It is suggested that both bacterial diversity and abundance of overall and/or individual bacterial populations are also of importance in regard to the GIT health of animals.

The comparison of excreta bacterial communities between pre-molting and immediate post-molting hens was also performed by PCoA based on the Jaccard distance. We found that excreta bacterial communities were significantly differentiated by forced molting (Figure 3). To explore the details 


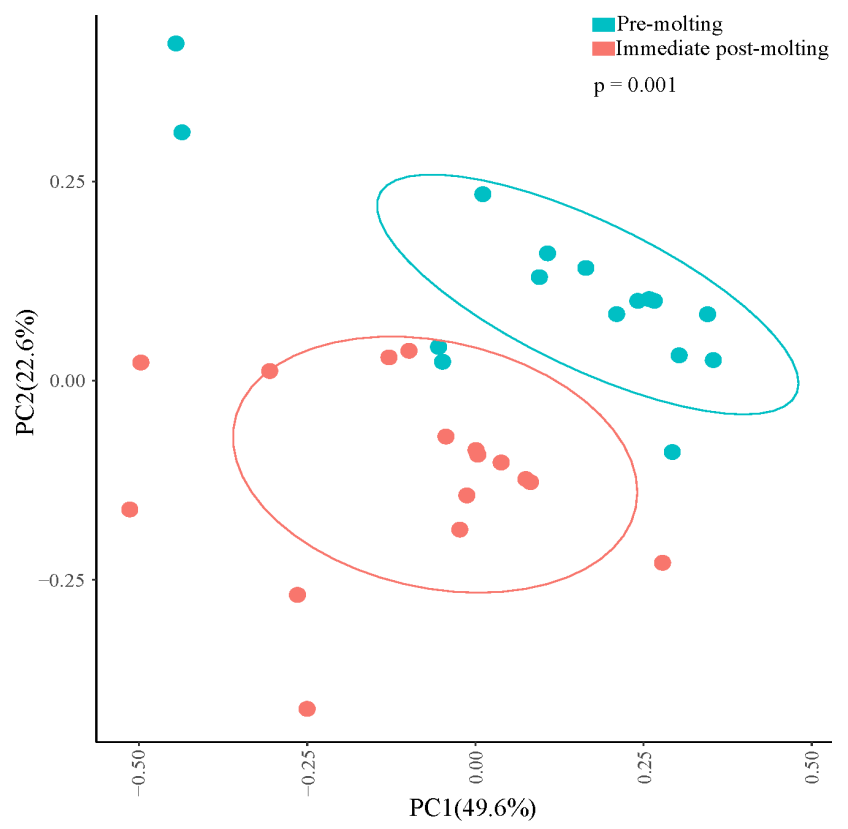

Figure 3. Jaccard distance based principal coordinate analysis $(\mathrm{PCOA})$ plot of pre-molting and immediate post-molting. The divergence of forced molting on excreta bacterial community was statistically tested by analysis of similarity (ANOSIM; permutations $=999$; ANOSIM's $r=0.236, p<0.001$ ). Percentages on the axes indicate variation explained by the principal coordinates of the jaccard distance and each symbol represents the bacterial community of each individual hen. Standard error ellipses represent $75 \%$ confidence areas.

\section{OTU Consensus taxonomy}

OTU10683 Vagococcus

OTU13201 Lactobacillus

OTU3333 Lactobacillus

OTU5941 Lactobacillus

OTU8946 Lactobacillus

OTU3617 Lactobacillus coleohominis

OTU10744 Lactobacillus helveticus

OTU2271 Lactobacillus helveticus

OTU7407 Lactobacillus reuteri

OTUT1418 5 Bacteroides

OTU2173 Bacteroides plebeius

OTU10570 Paraprevotellaceae

OTU11864 Paraprevotellaceae

OTU2835 Parabacteroides

OTU9239 Parabacteroides

OTU11308 Prevotella

OTU5683 $\$ 24-7$

OTU11852 Enterococcus cecorum

OTU4626 Leuconostocaceae

OTU6818 Streptococcus luteciae

OTU2773 Turicibacter

OTU2960 SMB53

OTU5590 Ruminococcus

OTU3992 Butyricicoccus pullicaecorum

OTU1459 Ruminococcaceae

OTU2281 Ruminococcaceae

OTU13248 Faecalibacterium prausnitzii

OTU6404 Faecalibacterium prausnitzii

OTU11185 Phascolarctobacterium

OTU1743 Clostridium saccharogumia

OTU15802 Escherichia coli

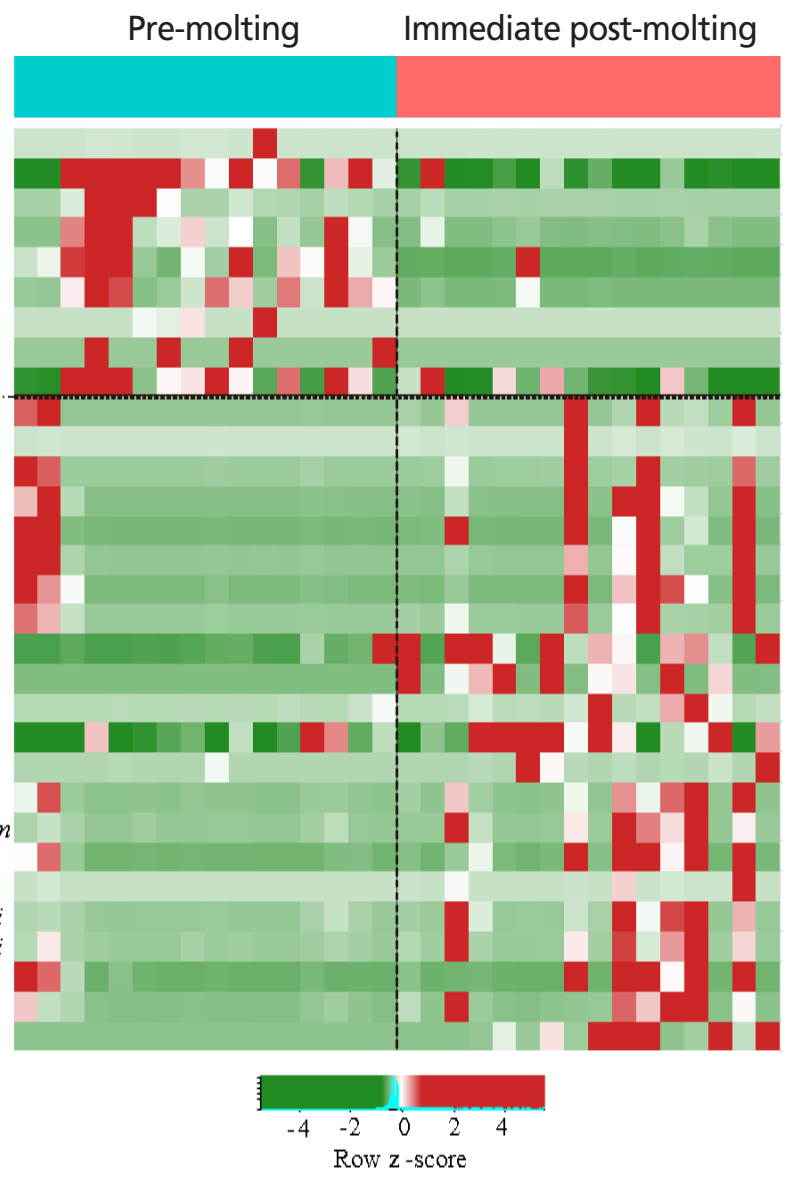

Figure 4. Heat map and logarithmic linear discriminant analysis scores of the differentially abundant bacteria between pre-molting and immediate-post molting hens determined by LEfSe analysis ( $a<0.05$, Kruskal-Wallis test). LEfSe, linear discriminant analysis effect size. 
on distinct excreta bacteria between groups, we performed LEfSe analysis. A total of 31 bacterial taxa were significantly discriminated in immediate post-molting hens (Figure 4). A total of 9 OTUs were more abundant in pre-molting hens, whereas 22 OTUs were enriched in post-molting hens (Table 2). In particular, Lactobacillus were dominated in pre-molting hens, whereas Bacteroides, Prevotella, Parabacteroides, Clostridium, Entercococcus, Streptococcus, and Escherichia were increased in post-molting hens. This result agrees with previous findings that the numbers of Lactobacilli in the crop were significantly decreased after forced molting with feed withdrawal [28]. A previous study has also shown an increased abundance of Enterobacteria, which is known as a pathogenic bacteria related to various inflammatory diseases in the GIT after withdrawing foods [26]. In addition, Boente et al [29] observed that Bacteroides and Parabacteroides strains were shown to be opportunistic pathogens, which suggests that forced molting of hens may lead to negative outcomes regarding pathogenic proliferation.

\section{CONCLUSION}

Microbial populations in the GIT are considerably altered by forced molting of hens with an increase in the diversity and variation of bacterial communities. Increasing colonization of pathogenic bacteria but decreasing colonization of Lactobacillus spp. in post-molting hens are identified, which indicates that the conventional molting procedure with feed withdrawal increases the susceptibility to pathogenic infections of aged laying hens. The result of this experiment improves our current understanding of microbial alterations by forced molting of aged laying hens and provides the opportunity to develop new dietary and management skills for improving performance and health of aged laying hens after molting.

Table 2. Excreta bacterial community differences between pre-molting and immediate post-molting hens using linear discriminant analysis effect size (LEfSe)

\begin{tabular}{|c|c|c|c|c|c|c|c|}
\hline Group & Phylum & Class & Order & Family & Genus & Species & $\begin{array}{c}\text { LDA score }^{1)} \\
\left(\log _{10}\right)\end{array}$ \\
\hline \multirow[t]{9}{*}{ Pre-molting } & Firmicutes & Bacilli & Lactobacillales & Lactobacillaceae & Lactobacillus & & 5.16 \\
\hline & Firmicutes & Bacilli & Lactobacillales & Lactobacillaceae & Lactobacillus & reuteri & 4.16 \\
\hline & Firmicutes & Bacilli & Lactobacillales & Enterococcaceae & Vagococcus & & 3.68 \\
\hline & Firmicutes & Bacilli & Lactobacillales & Lactobacillaceae & Lactobacillus & & 3.29 \\
\hline & Firmicutes & Bacilli & Lactobacillales & Lactobacillaceae & Lactobacillus & & 3.25 \\
\hline & Firmicutes & Bacilli & Lactobacillales & Lactobacillaceae & Lactobacillus & salivarius & 3.14 \\
\hline & Firmicutes & Bacilli & Lactobacillales & Lactobacillaceae & Lactobacillus & coleohominis & 3.11 \\
\hline & Firmicutes & Bacilli & Lactobacillales & Lactobacillaceae & Lactobacillus & & 3.10 \\
\hline & Firmicutes & Bacilli & Lactobacillales & Lactobacillaceae & Lactobacillus & & 3.01 \\
\hline \multirow[t]{23}{*}{ Post-molting } & Bacteroidetes & Bacteroidia & Bacteroidales & Paraprevotellaceae & & & 3.74 \\
\hline & Bacteroidetes & Bacteroidia & Bacteroidales & Bacteroidaceae & Bacteroides & plebeius & 3.61 \\
\hline & Bacteroidetes & Bacteroidia & Bacteroidales & Prevotellaceae & Prevotella & & 3.55 \\
\hline & Bacteroidetes & Bacteroidia & Bacteroidales & Paraprevotellaceae & & & 3.47 \\
\hline & Bacteroidetes & Bacteroidia & Bacteroidales & Bacteroidaceae & Bacteroides & & 3.39 \\
\hline & Bacteroidetes & Bacteroidia & Bacteroidales & S24_7 & & & 3.39 \\
\hline & Bacteroidetes & Bacteroidia & Bacteroidales & Porphyromonadaceae & Parabacteroides & & 3.30 \\
\hline & Bacteroidetes & Bacteroidia & Bacteroidales & Porphyromonadaceae & Parabacteroides & & 3.16 \\
\hline & Bacteroidetes & Bacteroidia & Bacteroidales & Bacteroidaceae & Bacteroides & & 3.05 \\
\hline & Firmicutes & Clostridia & Clostridiales & Lachnospiraceae & Ruminococcus & & 3.96 \\
\hline & Firmicutes & Clostridia & Clostridiales & Clostridiaceae & SMB53 & & 3.71 \\
\hline & Firmicutes & Clostridia & Clostridiales & Ruminococcaceae & & & 3.49 \\
\hline & Firmicutes & Clostridia & Clostridiales & Veillonellaceae & Phascolarctobacterium & & 3.44 \\
\hline & Firmicutes & Clostridia & Clostridiales & Ruminococcaceae & Butyricicoccus & pullicaecorum & 3.42 \\
\hline & Firmicutes & Clostridia & Clostridiales & Ruminococcaceae & & & 3.34 \\
\hline & Firmicutes & Clostridia & Clostridiales & Ruminococcaceae & Faecalibacterium & prausnitzii & 3.17 \\
\hline & Firmicutes & Clostridia & Clostridiales & Ruminococcaceae & Faecalibacterium & prausnitzii & 3.16 \\
\hline & Firmicutes & Erysipelotrichi & Erysipelotrichales & Erysipelotrichaceae & Clostridium & saccharogumia & 3.14 \\
\hline & Firmicutes & Bacilli & Lactobacillales & Enterococcaceae & Enterococcus & cecorum & 4.98 \\
\hline & Firmicutes & Bacilli & Lactobacillales & Leuconostocaceae & & & 3.74 \\
\hline & Firmicutes & Bacilli & Lactobacillales & Streptococcaceae & Streptococcus & luteciae & 3.68 \\
\hline & Firmicutes & Bacilli & Turicibacterales & Turicibacteraceae & Turicibacter & & 3.96 \\
\hline & Proteobacteria & Gammaproteobacteria & Enterobacteriales & Enterobacteriaceae & Escherichia & coli & 4.73 \\
\hline
\end{tabular}

\footnotetext{
1) Logarithmic linear discriminant analysis (LDA) scores of each discriminant bacterium (LDA>3.0).
} 


\section{CONFLICT OF INTEREST}

We certify that there is no conflict of interest with any financial organization regarding the material discussed in the manuscript.

\section{ACKNOWLEDGMENTS}

This research was carried out with the support of the Cooperative Research Program for Agriculture Science and Technology Development (Project No. PJ01256701), Rural Development Administration, Republic of Korea. This research was also supported by the Chung-Ang University Graduate Research Scholarship in 2018.

\section{REFERENCES}

1. Roberts JR. Factors affecting egg internal quality and egg shell quality in laying hens. J Poult Sci 2004;41:161-77. https://doi. org/10.2141/jpsa.41.161

2. Alodan MA, Mashaly MM. Effect of induced molting in laying hens on production and immune parameters. Poult Sci 1999; 78:171-7. https://doi.org/10.1093/ps/78.2.171

3. Patwardhan D, King A. Review: feed withdrawal and non feed withdrawal moult. World's Poult Sci J 2011;67:253-68. https://doi.org/10.1017/S0043933911000286

4. Ricke SC. The gastrointestinal tract ecology of Salmonella enteritidis colonization in molting hens. Poult Sci 2003;82: 1003-7. https://doi.org/10.1093/ps/82.6.1003

5. Oh JK, Pajarillo EAB, Chae JP, Kim IH, Kang DK. Protective effects of Bacillus subtilis against Salmonella infection in the microbiome of Hy-Line Brown layers. Asian-Australas J Anim Sci 2017;30:1332-9. https://doi.org/10.5713/ajas.17.0063

6. Wang J, Fan H, Han Y, Zhao J, Zhou Z. Characterization of the microbial communities along the gastrointestinal tract of sheep by 454 pyrosequencing analysis. Asian-Australas J Anim Sci 2017;30:100-10. https://doi.org/10.5713/ajas.16.0166

7. Hert DG, Fredlake CP, Barron AE. Advantages and limitations of next-generation sequencing technologies: A comparison of electrophoresis and non-electrophoresis methods. Electrophoresis 2008;29:4618-26. https://doi.org/10.1002/elps.2008 00456

8. Edgar RC. Search and clustering orders of magnitude faster than BLAST. Bioinformatics 2010;26:2460-1. https://doi.org/ 10.1093/bioinformatics/btq461

9. Gasperin C, Gamallo P, Agustini A, Lopes G, De Lima V. Using syntactic contexts for measuring word similarity. In: Proceedings of the Workshop on Semantic Knowledge Acquisition and Categorisation; 2001.

10. Segata N, Izard J, Waldron L, et al. Metagenomic biomarker discovery and explanation. Genome Biol 2011;12:R60. https:// doi.org/10.1186/gb-2011-12-6-r60
11. Kumar S, Stecher G, Tamura K. MEGA7: Molecular evolutionary genetics analysis version 7.0 for bigger datasets. Mol Biol Evol 2016;33:1870-4. https://doi.org/10.1093/molbev/ msw054

12. Videnska P, Rahman MM, Faldynova M, et al. Characterization of egg laying hen and broiler fecal microbiota in poultry farms in Croatia, Czech Republic, Hungary and Slovenia. PLoS One 2014;9:e110076. https://doi.org/10.1371/journal. pone. 0110076

13. Hamid H, Zhang JY, Li WX, et al. Interactions between the cecal microbiota and non-alcoholic steatohepatitis using laying hens as the model. Poult Sci 2019;98:2509-21. https://doi.org/ $10.3382 / \mathrm{ps} / \mathrm{pey} 596$

14. Videnska P, Faldynova M, Juricova $H$, et al. Chicken faecal microbiota and disturbances induced by single or repeated therapy with tetracycline and streptomycin. BMC Vet Res 2013;9:30. https://doi.org/10.1186/1746-6148-9-30

15. Dong XY, Azzam MMM, Zou XT. Effects of dietary threonine supplementation on intestinal barrier function and gut microbiota of laying hens. Poult Sci 2017;96:3654-63. https://doi. org/10.3382/ps/pex185

16. Turnbaugh PJ, Ley RE, Mahowald MA, et al. An obesity-associated gut microbiome with increased capacity for energy harvest. Nature 2006;444:1027-31. https://doi.org/10.1038/ nature 05414

17. Wong JMW, de Souza R, Kendall CWC, Emam A, Jenkins DJA. Colonic health: fermentation and short chain fatty acids. J Clin Gastroenterol 2006;40:235-43.

18. Yan W, Sun C, Yuan J, Yang N. Gut metagenomic analysis reveals prominent roles of Lactobacillus and cecal microbiota in chicken feed efficiency. Sci Rep 2017;7:45308. https://doi. org/10.1038/srep45308

19. Robinson EL, Thompson WL. Effect on weight gain of the addition of Lactobacillus acidophilus to the formula of newborn infants. J Pediatr 1952;41:395-8. https://doi.org/10.1016/ S0022-3476(52)80121-0

20. Angelakis E, Raoult D. The increase of Lactobacillus species in the gut flora of newborn broiler chicks and ducks is associated with weight gain. PLoS One 2010;5:e10463. https://doi. org/10.1371/journal.pone.0010463

21. Million M, Angelakis E, Paul M, Armougom F, Leibovici L, Raoult D. Comparative meta-analysis of the effect of Lactobacillus species on weight gain in humans and animals. Microb Pathog 2012;53:100-8. https://doi.org/10.1016/j.micpath.2012. 05.007

22. Dunkley KD, Callaway TR, Chalova VI, et al. Foodborne Salmonella ecology in the avian gastrointestinal tract. Anaerobe 2009;15:26-35. https://doi.org/10.1016/j.anaerobe.2008.05.007

23. Jin LZ, Ho YW, Ali AM, Abdullah N, Jalaludin S. Effect of adherent Lactobacillus spp. on in vitro adherence of salmonellae to the intestinal epithelial cells of chicken. J Appl Bacteriol 1996;81:201-6. https://doi.org/10.1111/j.1365-2672.1996. 
tb04501.x

24. Holt PS, Porter Jr RE. Microbiological and histopathological effects of an induced-molt fasting procedure on a Salmonella enteritidis infection in chickens. Avian Dis 1992;36:610-8.

25. Delaunay E, Abat C, Rolain JM. Enterococcus cecorum human infection, France. New Microbes New Infect 2015;7:50-1. https://doi.org/10.1016/j.nmni.2015.06.004

26. Remely M, Hippe B, Geretschlaeger I, Stegmayer S, Hoefinger I, Haslberger A. Increased gut microbiota diversity and abundance of Faecalibacterium prausnitzii and Akkermansia after fasting: a pilot study. Wien Klin Wochenschr 2015;127:3948. https://doi.org/10.1007/s00508-015-0755-1
27. Wang XJ, Feng JH, Zhang MH, Li XM, Ma DD, Chang SS. Effects of high ambient temperature on the community structure and composition of ileal microbiome of broilers. Poult Sci 2018;97:2153-8. https://doi.org/10.3382/ps/pey032

28. Durant JA, Corrier DE, Byrd JA, Stanker LH, Ricke SC. Feed deprivation affects crop environment and modulates Salmonella enteritidis colonization and invasion of Leghorn hens. Appl Environ Microbiol 1999;65:1919-23.

29. Boente RF, Ferreira LQ, Falcão LS, et al. Detection of resistance genes and susceptibility patterns in Bacteroides and Parabacteroides strains. Anaerobe 2010;16:190-4. https://doi.org/10. 1016/j.anaerobe.2010.02.003 